\title{
Why do patients decline amniocentesis? Analysis of factors influencing the decision to refuse invasive prenatal testing
}

Pawel Sadlecki, Marek Grabiec, Pawel Walentowicz and Malgorzata Walentowicz-Sadlecka

\begin{abstract}
Background: In recent years, determination of personalized risk for fetal chromosomal anomalies emerged as an important component of prenatal genetic counseling. Women in whom fetal risk for chromosomal aberrations is elevated are offered further testing. The aim of this study was to identify factors that may influence the decision to refuse invasive prenatal testing aimed at determination of fetal karyotype in a group of patients at increased risk of trisomy 21.

Methods: The analysis included 177 patients with singleton pregnancy, whose personalized risk score for trisomy 21 calculated on the basis of the combined test exceeded 1:300. Diagnostic amniocentesis was performed in 125 patients from this subset, since the remaining 52 women declined invasive prenatal testing. The following factors were analyzed as potential determinants of the decision to refuse amniocentesis: maternal age ( $\geq 35$ years), gravidity, number of miscarriages in previous pregnancies, educational status, marital status, indications to prenatal testing, gestational age at the time of prenatal testing, personalized risk score for fetal chromosomal aberrations and nuchal translucency (NT) value.
\end{abstract}

Results: A statistically significant relationship was found between the decision to refuse amniocentesis and the number of previous miscarriages, maternal educational level, NT values and personalized risk score for fetal chromosomal aberrations. Multivariate logistic regression analysis identified primary maternal education and history of more than two miscarriages as independent significant predictors of declining amniocentesis. Women with personalized risk scores for trisomy 21 greater than 1:100 opted out of invasive prenatal diagnosis significantly less often than the remaining participants.

Conclusion: In conclusion, the key role of high quality and accuracy of non-invasive diagnostic tests conducted in the first trimester should be emphasized as personalized risk score for fetal chromosomal aberrations determined based on their results is pivotal for further management of pregnancy. Equally important is to provide the patients with an accurate and comprehensible information about potential benefits and risks of invasive testing.

Keywords: Amniocetesis, Decision making, Trisomy 21, Combined test

\footnotetext{
* Correspondence: pawelsadlecki@wp.pl

Department of Obstetrics and Gynecology, Collegium Medicum in Bydgoszcz,

Nicolaus Copernicus University of Torun, ul. Ujejskiego 75, 85-168 Bydgoszcz,

Poland
}

(c) The Author(s). 2018 Open Access This article is distributed under the terms of the Creative Commons Attribution 4.0 International License (http://creativecommons.org/licenses/by/4.0/), which permits unrestricted use, distribution, and reproduction in any medium, provided you give appropriate credit to the original author(s) and the source, provide a link to the Creative Commons license, and indicate if changes were made. The Creative Commons Public Domain Dedication waiver (http://creativecommons.org/publicdomain/zero/1.0/) applies to the data made available in this article, unless otherwise stated. 


\section{Background}

Prenatal diagnosis of Down syndrome allows for informed decision-making with regard to pregnancy continuation or termination. A number of screening strategies for detection of prenatal trisomy 21 in the first and second trimester have been developed. In Polish pregnant women, the screening typically involves ultrasonographic measurement of nuchal translucency (NT) at 11-14 weeks of gestation, along with the determination of blood levels of free- $\beta$-human chorionic gonadotrophin $(\beta \mathrm{hCG})$ and pregnancy-associated plasma protein-A (PAPP-A) [1]. Based on the results of these tests, patient age and medical history, the so-called personalized risk score for fetal chromosomal aberrations is calculated. In line with the recommendations of the Polish Gynecological Society, the likelihood of fetal chromosomal aberration was considered high whenever the personalized risk score calculated on the basis of the combined test exceeded 1:300 [1]. The score determined on the basis of fetal NT at $11-13^{+6}$ weeks of gestation and maternal age provides approximately $75 \%$ sensitivity in detection of trisomy 21, and inclusion of PAPP-A level in the screening algorithm further increases its sensitivity, up to ca. 85-90\%, with only $5 \%$ false positive rate [2]. If the results of the screening point to an increased risk of fetal chromosomal aberrations, invasive testing, such as amniocentesis or trophoblast biopsy, is offered to pregnant women to determine fetal karyotype. However, the invasive tests are associated with increased risk of pregnancy complications, including miscarriage [3]. The aim of this study was to identify factors that may influence the decision to refuse invasive prenatal testing aimed at determination of fetal karyotype in a group of patients at increased risk of trisomy 21.

\section{Methods}

A total of 2251 patients have been tested for chromosomal aberrations and fetal defects at the Prenatal Genetic Unit, Department of Gynecology, Obstetrics and Oncologic Gynecology, Nicolaus Copernicus University, Collegium Medicum in Bydgoszcz in 2014. A total of 2002 combined tests were conducted to calculate personalized risk score for fetal chromosomal aberrations on the basis of ultrasonographic markers NT, nasal bone (NB) at $45-84 \mathrm{~mm}$ CRL, serum levels of $\beta$-hCG and PAPP-A, in line with the recommendations of the Fetal Medicine Foundation (FMF) and Polish Gynecological Society $[1,2]$. Some eligible patients refused to participate in the first trimester screening carried out at our Department; unfortunately, we were unable to estimate the size of this subgroup. Patients' data were extracted from their medical histories obtained by two physicians specialized in medical genetics, employees of our
Department. Both of them were trained in prenatal testing, and conducted a 20-min interview with each patient participating in the screening. Two counseling sessions were scheduled, one prior to non-invasive screening, and another one after the results were available (usually after 2 weeks). During the second session, the patients were advised if the invasive screening was required or not. Total number of diagnostic amniocenteses performed in 2014 was 202; aside from increased risk of fetal anomalies, other indications to invasive prenatal testing included maternal age $>35$ years, history of chromosomal aberration or fetal defect in previous pregnancy, and abnormal result of the triple screen test. Eventually, the analysis included 177 patients with singleton pregnancy, whose personalized risk score for trisomy 21 calculated on the basis of the combined test exceeded 1 : 300. Diagnostic amniocentesis was performed in 125 patients from this subset, since the remaining 52 women declined invasive prenatal testing. The following factors were analyzed as potential determinants of the decision to refuse amniocentesis: maternal age ( $\geq 35$ years), gravidity, number of miscarriages in previous pregnancies, educational status, marital status, indications to prenatal testing, gestational age at the time of prenatal testing, personalized risk score for fetal chromosomal aberrations and NT value. The protocol of the study was approved by the Local Bioethics Committee at Collegium Medicum in Bydgoszcz, Nicolaus Copernicus University in Torun (decision no. KB 239/2011), and written informed consent was sought from all the study participants. Statistical analysis of the results was carried out with PQStat ver. 1.6 package. Association between declining amniocentesis and explanatory variables were verified with chi $^{\wedge}$ test. Moreover, all explanatory variables were tested as potential predictors of declining invasive prenatal diagnosis with univariate and multivariate logistic regression models. The relationships were considered significant at $p<0.05$ and highly significant at $p<0.01$.

\section{Results}

A statistically significant relationship $\left(\mathrm{chi}^{\wedge} 2=7.3417, \mathrm{df}=2\right.$, $p=0.0254$ ) was found between the decision to refuse amniocentesis and the number of previous miscarriages. Women with a history of two or more miscarriages in previous pregnancies declined invasive prenatal testing significantly more often than the other patients (Fig. 1).

The decision to refuse amniocentesis correlated significantly with maternal educational level $\left(\operatorname{chi}^{\wedge} 2=8\right.$. $6208, \mathrm{df}=3, p=0.0348$ ). Women with primary education declined invasive prenatal testing most often of all the study subjects (Fig. 2).

A statistically significant relationship $\left(\mathrm{chi}^{\wedge} 2=6.1364\right.$, $\mathrm{df}=1, p=0.0132$ ) was observed between declining invasive prenatal diagnosis and nuchal translucency (NT) 


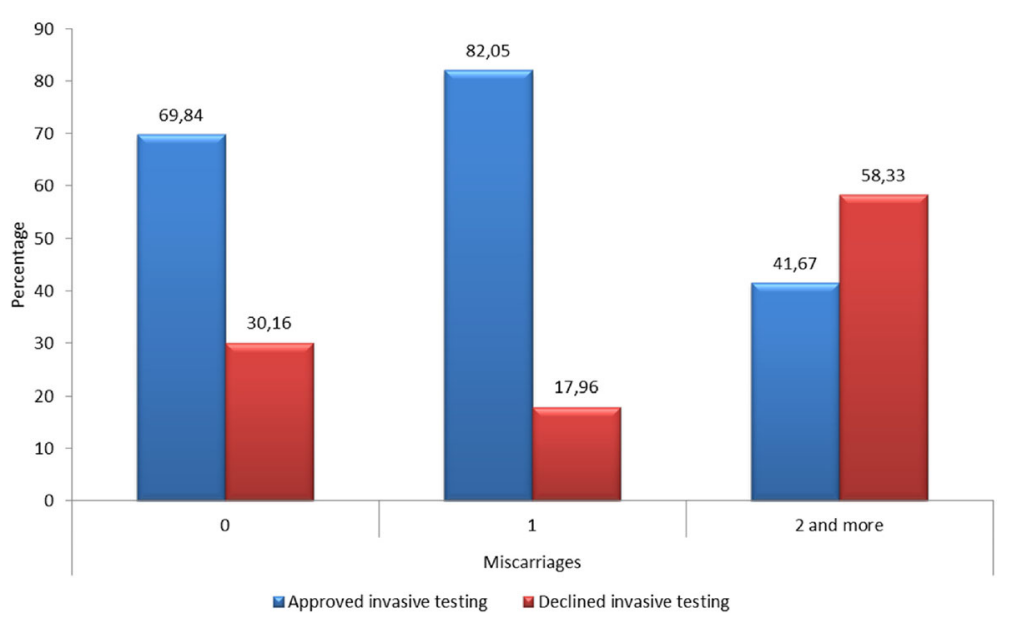

Fig. 1 Distribution according to the number of miscarriages. Distribution of patients who declined amniocentesis according to the number of miscarriages in previous pregnancies $(p=0.0254)$

values. Patients whose fetuses presented with $\mathrm{NT}<2.5 \mathrm{~mm}$ refused amniocentesis significantly more often than the other women (Fig. 3).

A highly significant association $\left(\mathrm{chi}^{\wedge} 2=9.6566, \mathrm{df}=2\right.$, $p=0.0080)$ was found between declining amniocentesis and personalized risk score for fetal chromosomal aberrations. The lower the personalized risk score for trisomy 21, the more often pregnant women refused prenatal invasive testing (Fig. 4).

The decision to refuse prenatal invasive testing did not correlate significantly with maternal age $(<35$ years vs. $\geq$ 35 years; $p=0.94)$, gravidity $(<2$ vs. $\geq 3 ; p=0.99)$, marital status (married vs. others; $p=0.27$ ) and gestational age at the time of the combined test (11 vs. 12 vs. $13 ; p=0.42$ ).

Logistic regression models to predict the decision to refuse amniocentesis on the basis of analyzed explanatory variables are presented in Table 1.
Multivariate logistic regression analysis identified primary maternal education and history of more than two miscarriages as independent significant predictors of declining amniocentesis. Women with personalized risk scores for trisomy 21 greater than 1:100 opted out of invasive prenatal diagnosis significantly less often than the remaining participants.

\section{Discussion}

The accuracy of prenatal cytogenetic diagnostic tests for fetal chromosomal anomalies has been confirmed over the last 40 years. Second trimester amniocentesis is the most commonly performed prenatal invasive diagnostic procedure. In recent years, determination of personalized risk for fetal chromosomal anomalies emerged as an important component of prenatal genetic counseling [4]. Women in whom fetal risk for chromosomal aberrations

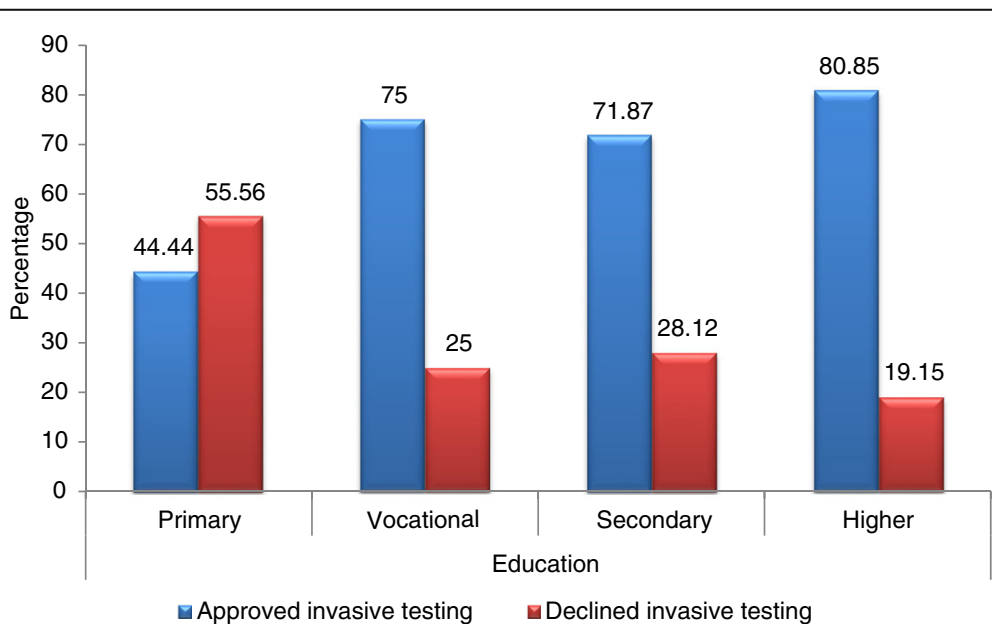

Fig. 2 Distribution according to the educational level. Distribution of patients who declined amniocentesis according to their educational level ( $p=0.0348)$ 


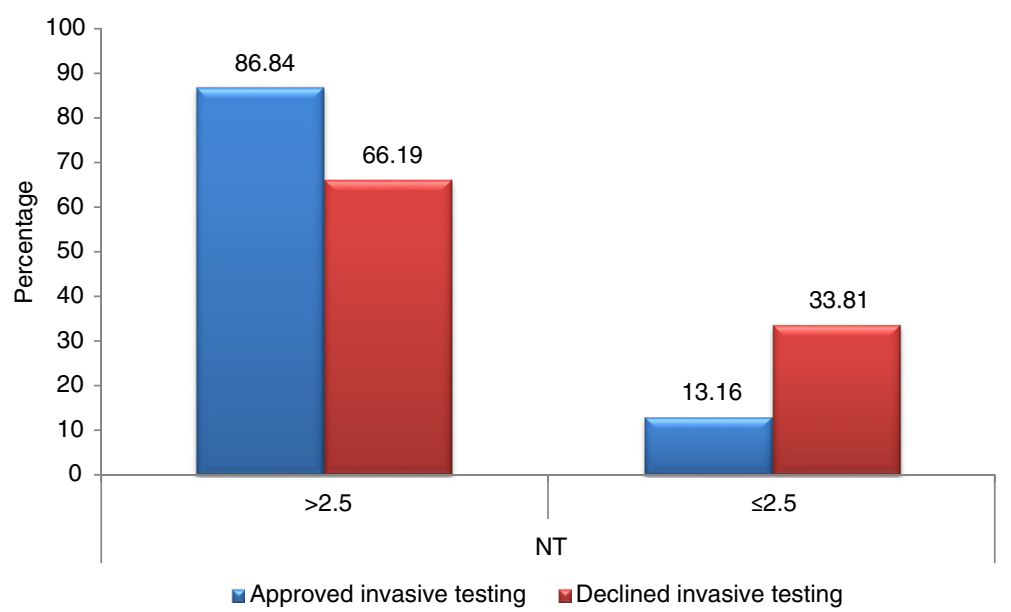

Fig. 3 Distribution according to the nuchal translucency. Distribution of patients who declined amniocentesis according to nuchal translucency (NT) values $(p=0.0132)$

is elevated are offered further testing. However, the decision regarding invasive prenatal diagnosis needs to be made within tight timeframes since the window of gestational age during which such tests can be performed safely and provide accurate results is quite narrow [5]. Previous studies revealed that many women refer to prenatal screening and testing appointments with little understanding about the nature of the exams $[5,6]$. Parents should understand the nature of the screening choices they have been offered; however, literature data imply that they may do not receive adequate, timely information from health professionals and thus are unable to make an informed decision [6]. The information and advice provided by an obstetrician prior to referral to a genetics center is likely an important determinant of maternal decision to undergo amniocentesis [7]. In our opinion, our study should be considered unique since it was conducted in Poland, whereas most similar previous studies were carried out in the United States or in Western Europe. To the best of our knowledge, the number of studies including subjects from Eastern Europe who refused invasive prenatal testing despite medical indications, is limited. We analyzed medical records of $177 \mathrm{pa}-$ tients qualified for amniocentesis, selected from a group of 2251 pregnant women tested for chromosomal aberrations and fetal defects. Nearly $30 \%$ of women from the study group refused invasive testing although their risk score for trisomy 21 exceeded 1:300; this fraction should be considered high. Other 125 patients underwent diagnostic amniocentesis. In the study conducted by Kuppermann et al., amniocentesis was carried out in 43 out of 710 women [8]. In our opinion, it is vitally important for

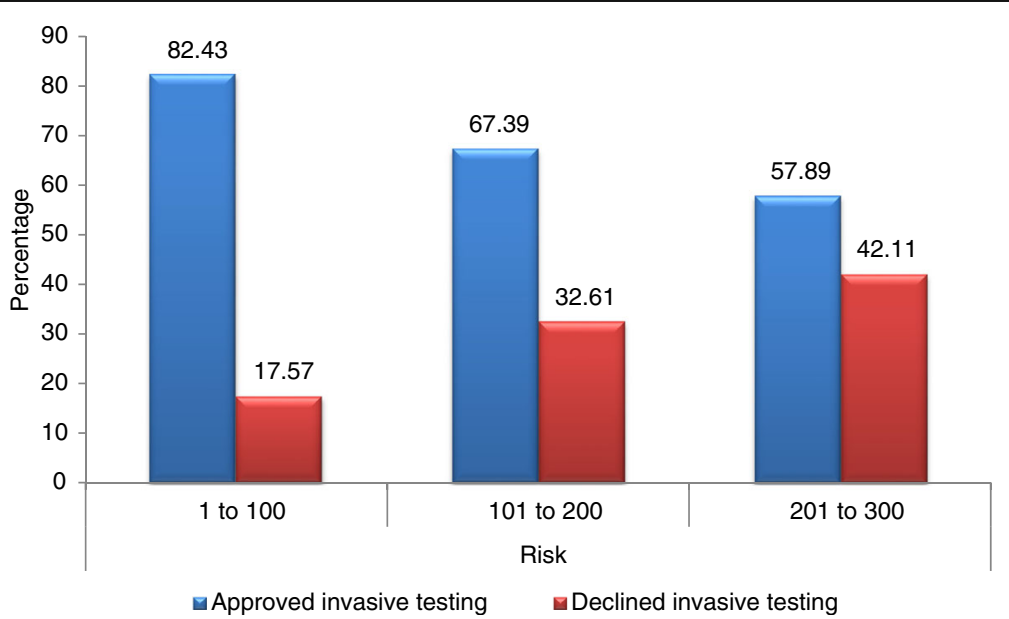

Fig. 4 Distribution according to the personalized risk scores. Distribution of patients who declined amniocentesis according to their personalized risk scores for trisomy $21(p=0.0080)$ 
Table 1 Logistic regression models to predict the decision to refuse amniocentesis on the basis of analyzed explanatory variables

\begin{tabular}{|c|c|c|c|c|c|}
\hline & b coefficient & $p$-value & Odds ratio & $-95 \% \mathrm{Cl}$ & $+95 \% \mathrm{Cl}$ \\
\hline Intercept & -0.894701 & 0.000525 & 0.40873 & 0.246506 & 0.677711 \\
\hline Primary education & 1.312228 & 0.014799 & 3.714441 & 1.293011 & 10.6705 \\
\hline Miscarriages> 2 & 1.796904 & 0.025365 & 6.030948 & 1.248176 & 29.140396 \\
\hline Risk score > 1:100 & -0.937951 & 0.027392 & 0.391429 & 0.170106 & 0.900716 \\
\hline
\end{tabular}

pregnant women to understand the purpose and potential consequences of prenatal testing, especially that many patients seem to perceive non-invasive prenatal tests merely as a routine component of a larger laboratory workup $[8,9]$.

In our study, the decision to refuse amniocentesis was made most often by women with primary education; furthermore, primary education turned out to be the only independent predictor of declining invasive prenatal diagnosis on multivariate logistic regression analysis. However, this does not seem to be a universal rule since according to McCoyd, even relatively well-educated and economically stable persons may present with a surprising lack of knowledge and understanding on the purpose of the exam and possibility of fetal diagnosis [10]. During perinatal period, women are particularly vulnerable to anxiety and depressive disorders, and potential problems with their fetuses seem to contribute significantly to this conditions [11]. Patients in whom screening tests showed increased risk for fetal anomaly or revealed an actual defect, are typically devastated and confused [12]. The vast majority of pregnant women do not consider potential problems with their fetuses. Even upon referral to a medical appointment aimed at fetal health assessment, only a small proportion of patients discuss potential risk for a fetal anomaly with their partner or anyone else [9]. This implies that pregnant women display a relatively high level of denial or are just not prepared for possible diagnosis of fetal anomaly. This may explain why upon such diagnosis women are truly shocked and have a sense of betrayal, rather than being "only" surprised or saddened [8]. Pregnancy planning is just one indicator of preparedness for a gestation; the percentage of planned pregnancies in the United States is estimated at 50\% [10]. Preparation for pregnancy can be considered a valuable resource for decision-making, especially in a crisis situation, such as detection of a fetal anomaly. Available evidence suggests that under such circumstances, women with unplanned pregnancies may be more prone to decision-making problems [13, 14]. The principal reasons behind undergoing prenatal tests are reassurance and the desire of knowledge about the fetal health. The decision to decline prenatal testing may be driven by personal views on pregnancy termination and the fear for iatrogenic pregnancy loss [13]. Although amniocentesis is associated with an increased risk for miscarriage, it should be stressed that the exact risk has still not been determined and its available estimates vary from $1: 100$ to $1: 1600$ [15]. In this study, women with a history of at least two miscarriages in previous pregnancies refused invasive prenatal diagnosis significantly more often than other subjects. Furthermore, the history of at least two miscarriages turned out to be an independent significant predictor of declining amniocentesis. The obstetric history of a woman is likely an important determinant of her decision to approve/refuse an invasive procedure [16]. Surprisingly, however, Sharda et al. showed that nearly $50 \%$ of women with unfavorable obstetric history and ultrasonographic evidence of soft aneuploidy markers agreed to take risk of amniocentesis rather than having an abnormal child. On the other hand, some women with two or more live children (including one with a genetic disorder) and primigravidas were reluctant to undergo an invasive procedure [16]. Still little is known on the factors that influence maternal decision to accept or decline diagnostic amniocentesis, and most of available data in this matter are not evidence-based or reflect solely personal experiences [17]. Potential determinants of the decision to undergo/ refuse invasive prenatal testing include the method of conception, age, parity, consanguinity, family history of congenital anomalies, history of miscarriage, twin gestation, socioeconomic background and religion. In our series, declining amniocentesis did not correlate significantly with maternal age ( $<35$ years vs. $\geq 35$ years), gravidity ( $<2$ vs. $\geq 3$ ), marital status (married vs.others) and gestational age at the time of non-invasivescreening (11 vs. 12 vs. 13). Many women who conceived via assisted reproduction technology (ART) are older than 35 years, either due to previous unsuccessful attempts to get pregnant or as a consequence of conscious decision to delay childbearing [18]. Women who undergo in vitro fertilization (IVF) may theoretically be reluctant to diagnostic amniocentesis owing attributed risk of miscarriage. However, some of them may seek additional assurance that their pregnancies are karyotypically normal, owing past history of infertility. How these conflicting factors influencematernal decision to approve/decline prenatal testing is largely unknown [18]. Nevertheless, in our study, all pregnant women with a history of ART procedures and high personalized risk scores for trisomy $21(N=7)$ opted for an invasive 
testing. Also the patients whose fetuses presented with nuchal translucency values $>2.5 \mathrm{~mm}$ and those with personalized risk scores for trisomy 21 greater than 1:100 refused amniocentesis significantly less often than the remaining participants of this study. Patients who did not give their consent for invasive tests can be offered non-invasive prenatal testing (NIPT) [19, 20]. Rapid advances in NIPT based on cell-free fetal DNA in maternal plasma have now made it possible to identify pregnancies affected by trisomy 21 from 10 weeks of gestation; the blood test provides high accuracy (>99\%) and low false-positive rate $(0.1 \%)$ in identification of affected pregnancies [21]. NIPT has two key clinical advantages over invasive testing: it does not pose a risk of miscarriage and can be conducted early in pregnancy. However, it should be remembered that NIPT is not currently considered fully diagnostic, and therefore, its positive result needs to be verified by means of an invasive testing [22]. Widespread use of fetal cell-free DNA testing would with no doubt reduce the number of unnecessary invasive tests (amniocentesis and chorionic villi sampling) and eliminate associated risk for miscarriage [23]. Moreover, the use of NIPT may exert a salient effect on pregnant woman's general attitude to prenatal genetic testing. Many patients who would currently decline prenatal genetic testing would likely opt for performing NIPT [24].

\section{Conclusion}

In conclusion, the key role of high quality and accuracy of non-invasive diagnostic tests conducted in the first trimester should be emphasized as personalized risk score for fetal chromosomal aberrations determined based on their results is pivotal for further management of pregnancy. Equally important is to provide the patients with an accurate and comprehensible information about potential benefits and risks of invasive testing. In our opinion, patients' educational level may influence their decisions regarding testing, and thus, all pregnant women should be provided with a clear information about available prenatal diagnostic options and their outcomes. Furthermore, it should be remembered that a subset of patients who declined invasive prenatal testing still may give their consent for NIPT.

\section{Abbreviations}

ART: Assisted reproduction technology; CRL: Crown rump length; FMF: Fetal Medicine Foundation; IVF: In vitro fertilization; NB: Nasal bone; NIPT: Noninvasive prenatal testing; NT: Nuchal translucency; PAPP-A: Pregnancy associated plasma protein - A; $\beta$ hCG: Free $\beta$ - human chorionic gonadotropin

\section{Availability of data and materials}

The data that support the findings of this study are available from the authors upon reasonable request.

\section{Authors' contributions}

PS,MWS, MG designed the study. PS, PW executed the study. PS wrote the first draft of the manuscript. PS, MWS, MG participated in data collection and analysis. PS, MWS, MG, PW, approved the final version of the manuscript.

\section{Ethics approval and consent to participate}

The protocol of the study was approved by the Local Bioethics Committee at Collegium Medicum in Bydgoszcz, Nicolaus Copernicus University in Torun (decision no. KB 239/2011), and written informed consent was sought from all the study participants.

\section{Competing interests}

The authors declare that they have no competing interests.

\section{Publisher's Note}

Springer Nature remains neutral with regard to jurisdictional claims in published maps and institutional affiliations.

Received: 13 July 2016 Accepted: 30 April 2018

Published online: 16 May 2018

\section{References}

1. Kotarski J, et al. Rekomendacje Polskiego Towarzystwa Ginekologicznego dotyczace postępowania w zakresie diagnostyki prenatalnej Ginekol Pol. Ginekol Pol. 2009;80:390-3.

2. Snijders R, Noble P, Serbie N, et al. UK multicentre project on assessment of risk of trisomy 21 by maternal age and fetal nuchal-translucency thickness at 10-14 weeks of gestation. Fetal Medicine Foundation First Trimester Screenig Group. Lancet. 1998;351:343-6.

3. Cederholm M, Haglund B, Axelsson O. Maternal complications following amniocentesis and chorionic villus sampling for prenatal karyotyping. BJOG. 2003;110(4):392-9

4. Tseng JJ, Chou MM, Lo FC, Lai HY, Chen MH, Ho ES. Detection of chromosome aberrations in the second trimester using genetic amniocentesis: experience during 1995-2004. Taiwan J Obstet Gynecol. 2006:45:39-41.

5. Ivry T. At the back stage of prenatal care: Japanese Ob-Gyns negotiating prenatal diagnosis. Med Anthropol Quar. 2006;20:441-68.

6. Nicols M. Vulnerability of first-time expectant mothers during ultrasound scans: an evaluation of the external pressures that influence the process of informed choice. Health Care Women Int. 2007;28:525-33.

7. Skirton $\mathrm{H}, \mathrm{Barr} \mathrm{O}$. Antenatal screening and informed choice: a cross-sectional survey of parents and professionals. Midwifery. 2010;26(6):596-602.

8. Kuppermann M, Pena S, Bishop JT, Nakagawa S, Gregorich SE, Sit A, Vargas J, Caughey AB, Sykes S, Pierce L, Norton ME. Effect of enhanced information, values clarification, and removal of financial barriers on use of prenatal genetic testing: a randomized clinical trial. JAMA. 2014;312(12):1210-7. https://doi.org/ 10.1001/jama.2014.11479.

9. Norton ME, Kuppermann M. Women should decide which conditions matter. Am J Obstet Gynecol. 2016;215(5):583-587.e1. https://doi.org/10. 1016/j.ajog.2016.06.045.

10. McCoyd J. Preparation for prenatal decision-making: a baseline of knowledge and reflection in women participating in prenatal screening. J Psychosom Obstet Gynaecol. 2013;34(1):3-8.

11. Sanhal CY, Mendilcioglu I, Ozekinci M, Simsek M, Bozkurt S. Comparison of pre-procedural anxiety and depression scores for patients undergoing chorion villus sampling and amniocentesis: an alternative perspective on prenatal invasive techniques. Pak J Med Sci. 2015;31(5):1038-42.

12. Korenromp MJ, Page-Chirstiaens GCML, van den Bout J, et al. Psychological consequences of termination of pregnancy for fetal anomaly: similarities and differences between partners. Prenat Diagn. 2005;25:1226-33.

13. Van den Berg M, Timmermans DR, Kleinveld JH, Garcia E, van Vugt JM, van der Wal G. Accepting or declining the offer of prenatal screening for congenital defects: test uptake and women's reasons. Prenat Diagn. 2005;25:84-90.

14. Chard RL, Norton ME. Genetic counseling for patients considering screening and diagnosis for chromosomal abnormalities. Clin Lab Med. 2016;36(2): 227-36. https://doi.org/10.1016/j.cll.2016.01.005.

15. Odibo AO, Gray DL, Dicke JM, Stamilio DM, Macones GA, Crane JP. Revisiting the fetal loss rate after second-trimester genetic amniocentesis: a single center's 16-year experience. Obstet Gynecol. 2008;111:589-95. 
16. Sharda S, Phadke SR. Uptake of invasive prenatal diagnostic tests in women after detection of soft markers for chromosomal abnormality on ultrasonographic evaluation. J Perinatol. 2007;27:550-5.

17. Johnson K, Kelley J, Saxton V, Walker SP, Hui L. Declining invasive prenatal diagnostic procedures: a comparison of tertiary hospital and national data from 2012 to 2015. Aust N Z J Obstet Gynaecol. 2017;57(2):152-6. https://doi.org/10.1111/ajo.12590.

18. Ragni G, Mosconi P, Baldini MP, Somigliana E, Vegetti W, Caliari I, Nicolosi AE. Health-related quality of life and need for IVF in 1000 Italian infertile couples. Hum Reprod. 2005;20:1286-91.

19. Wang $T$, et al. Detection of complex deletions in chromosomes 13 and 21 in a fetus by noninvasive prenatal testing. Mol Cytogenet. 2016;9:3.

20. Shethet a. Prenatal screening of cytogenetic anomalies - a western Indian experience. BMC Pregnancy Childb. 2015;15:90,

21. Nicolaides KH, Syngelaki A, Ashoor G, Birdir C, Touzet G Noninvasive prenatal testing for fetal trisomies in a routinely screened first-trimester population. Am J Obstet Gynecol. 2012; 207(5): 374:e 1-6. https://doi.org/10. 1016/j.ajog.2012.08.033.

22. Gregg AR, Gross SJ, Best RG, Monaghan KG, Bajaj K, Skotko BG, et al. ACMG statement on noninvasive prenatal screening for fetal aneuploidy. Genet Med. 2013;15:395-8.

23. Nicolaides $\mathrm{KH}$. Validation of targeted sequencing of single-nucleotide polymorphism for non-invasive prenatal detection of aneuploidy of chromosomes 13, 18, 21, X and Y. Prenat Diag. 2013;33:575-9.

24. Nishiyama M, Sekizawa A, Ogawa K, Sawai H, Nakamura H, Samura O, et al. Factors affecting parental decisions to terminate pregnancy in the presence of chromosome abnormalities: a Japanese multicenter study. Prenat Diagn. 2016;36(12):1121-6. https://doi.org/10.1002/pd.4947.

\section{Ready to submit your research? Choose BMC and benefit from:}

- fast, convenient online submission

- thorough peer review by experienced researchers in your field

- rapid publication on acceptance

- support for research data, including large and complex data types

- gold Open Access which fosters wider collaboration and increased citations

- maximum visibility for your research: over $100 \mathrm{M}$ website views per year 